\title{
1 3D Printing of five-in-one dose combination polypill with defined immediate and
}

\section{2 sustained release profiles}

3 Shaban A. Khaled ${ }^{a}$, Jonathan C. Burley ${ }^{a}$, Morgan R. Alexander ${ }^{a}$, Jing Yang ${ }^{b}$ and Clive J. Roberts ${ }^{a^{*}}$

4 a'Laboratory of Biophysics and Surface Analysis, School of Pharmacy, The University of Nottingham,

$5 \quad$ Nottingham, NG7 2RD, UK

$6{ }^{b}$ Division of Drug Delivery and Tissue Engineering, School of Pharmacy, The University of

7 Nottingham, Nottingham, NG7 2RD, UK

$8 \quad$ *Correspondence to: Clive J Roberts

9 Address: School of Pharmacy, The University of Nottingham, University Park, NG7 2RD, UK

10 Tel: +441159515048

11 Fax: +44 1159515102

12 Email: clive.roberts@nottingham.ac.uk 


\section{Abstract}

22 We have used three dimensional (3D) extrusion printing to manufacture a multi-active solid dosage 23 form or so called polypill. This contains five compartmentalised drugs with two independently 24 controlled and well-defined release profiles. This polypill demonstrates that complex medication 25 regimes can be combined in a single personalised tablet. This could potentially improve adherence for 26 those patients currently taking many separate tablets and also allow ready tailoring of a particular drug 27 combination/drug release for the needs of an individual. The polypill here represents a cardiovascular treatment regime with the incorporation of an immediate release compartment with aspirin and hydrochlorothiazide and three sustained release compartments containing pravastatin, atenolol, and ramipril. X-ray powder diffraction (XRPD) and Attenuated Total Reflectance Fourier Transform Infrared Spectroscopy (ATR-FTIR) were used to assess drug-excipient interaction. The printed polypills were evaluated for drug release using USP dissolution testing. We found that the polypill showed the intended immediate and sustained release profiles based upon the active/excipient ratio used.

\section{Keywords}

36 3D printing; Polypill; Sustained release; Immediate release; Personalised medicine

\section{Chemical compounds studied in this article}

38 Aspirin (PubChem CID: 2244); Hydrochlorothiazide (PubChem CID: 3639); Atenolol (PubChem 


\section{Introduction}

43 The use of multiple medications to control complex diseases such as cancer and heart diseases is an increasingly used therapeutic strategy [1,2]. Each active pharmaceutical ingredient is traditionally administered via a separate dosage form [2]. This is inconvenient, can lead to errors in medication and presents significant patient compliance issues [2,3]. Combining multiple actives into a single tablet with appropriate release profiles and doses (potentially optimised for individuals) is an attractive alternative [3-5].

The term "polypill" refers to a tablet that is composed of a combination of several medicines [5], and has been previously studied as a concept to treat and prevent cardiovascular disease and high blood pressure [6-9]. This polypill (in fact a capsule) manufactured by Cadila Pharmaceuticals Limited under trade name of Polycap ${ }^{\mathrm{TM}}$ is the only polypill formulation commercially available $[7,8,10]$. Cardiovascular disease is the most common cause of death globally and requires managing as a chronic condition in many people during large portions of their lifetime [11]. Based on previous work, we suggest that additive manufacturing or $3 \mathrm{D}$ printing is potentially well suited to producing a multicomponent polypill formulation $[4,8,9]$. As an approach 3D printing also offers the opportunity to produce personalised medicines and is adaptable to a distributed manufacturing model [4]. The freedom to form specific geometries in comparison to the restrictions of traditional tableting via powder compression can be used to separate incompatible substances and to enable different release rates using shape and size as well as excipient manipulation $[4,12]$. Here we have designed a 5component polypill based upon the currently available "polycap" commercial formulation with three sustained release compartments containing pravastatin, atenolol, and ramipril, which were physically separated by a hydrophobic cellulose acetate shell designed to act as a permeable carrier, and covered with an immediate release aspirin and hydrochlorothiazide compartment (Fig. 1.). Atenolol is a betablocker agent which is used to treat hypertension and also prevent and/or treat heart attack [13]. Hydrochlorothiazide is a thiazide diuretic used to prevent absorption of too much salt and to treat oedema or fluid retention in individuals with congestive heart failure, kidney disorder, and liver 
cirrhosis [14]. Ramipril is an angiotensin converting enzyme (ACE) used for treatment of 69 hypertension and congestive heart failure which improves heart function after a heart attack [15]. Aspirin is an antiplatelet used to reduce the risk of blood clotting and reduce heart attacks or strokes [16]. Pravastatin is a 3-hydroxy-3-methylglutaryl-coenzyme A (HMG-CoA) reductase inhibitors used to reduce blood cholesterol and triglycerides in hyperlipidaemic patients and lower rates of strokes and heart attacks [17].

$3 \mathrm{D}$ printing is a process used to fabricate $3 \mathrm{D}$ objects by laying down successive material layers in different shapes taken directly from a digital file [18]. There has been a significant recent growth in interest of 3D printing as a tool in pharmaceutics and personalised medicine [19-23]. For example, a heat based fused deposition modelling 3D printer $\left(>200{ }^{\circ} \mathrm{C}\right.$ ) has been used to extrude 5aminosalicylic acid (5-ASA, mesalazine), and 4-aminosalicylic acid (4-ASA) and prednisolone loaded poly (vinyl alcohol) (PVA) filaments and produce simple solid tablets [19, 24]. However, this approach would not be suitable generally due to the possibility of heat induced degradation of thermally sensitive drugs. Also, there are not many reports of printing a single drug formulation with multiple release mechanisms $[4,25]$. Katstra et al. employed multi-steps $3 \mathrm{D}$ printing to deposit chlorphenaramin maleate (antihistamine used in the prevention of symptoms of allergic conditions such as rhinitis and urticaria) as a binder onto powdered excipients (the amount of drug deposited was $5.45 \mathrm{mg})[25,26]$. However, issues such as ink bleeding, migration, and capillary effect due to drug/binder oversaturation are difficult to avoid for printing of larger drugs doses such as $500 \mathrm{mg}$ of paracetamol or ibuprofen $[25,26]$. Problems with this approach include long drying times (in excess of 50 hours) and high friability (>1\%) of the resultant tablet $[25,26]$.

To address the above mentioned issues of drug degradation and the complexity in published 3D printing processes we have employed a 3D extrusion system operated at room-temperature to manufacture a polypill capable of delivering the five drugs via two predictable release mechanisms. A hydrophobic cellulose acetate shell was first extruded then the active drugs atenolol, pravastatin, and ramipril were mixed with a hydrophilic matrix (HPMC) and extruded in to the segmented 
compartments of cellulose acetate to form sustained release compartments. Aspirin and hydrochlorothiazide were mixed with a disintegrant; sodium starch glycolate and other excipients and extruded directly on the top of the sustained release compartments, to give an immediate release compartment. A series of raised dots were also printed onto the top of the tablet to facilitate identification of the formulation both visually and by touch, the composition of these was the same as the upper "immediate release" layer. The printed tablets were tested for drug release and drugexcipients interaction using United States Pharmacopeia Convention (USP) Type I apparatus dissolution tester, X-Ray Powder Diffraction (XRPD), and Attenuated Total Reflectance Fourier Transform Infrared Spectroscopy (ATR-FTIR).

\section{Materials and methods}

\subsection{Materials}

Ramipril and pravastatin sodium were supplied by Kemprotec Limited (Cumbria, UK). Atenolol, aspirin, and hydrochlorothiazide, polyvinylpyrrolidine (PVP) and lactose were supplied by SigmaAldrich (Gillingham, UK). D-mannitol $99 \%$ was purchased from VWR International Ltd. (Leicestershire, UK). Sodium starch glycolate (Primojel ${ }^{\circledR}$ ) was kindly supplied as a gift from DFE Pharma. Hydroxypropyl methylcelloluse (HPMC K100M CR) (Methocel TM) was a gift from Colorcon $^{\circledR}$. Milli-Q water (resistivity $18.2 \mathrm{M} \Omega \mathrm{cm}$ ) was used for all formulations and solutions. All other reagents were of either HPLC or analytical grade.

\subsection{Methods}

\subsubsection{Design of polypill}

A segmented tablet strategy was chosen to ensure that the actives were separated and could achieve the desired independent control of their release (Fig. 1). This concept provides flexibility in production of a 3D printed polypill with tunable drug release based on modifying the drug loading and excipient composition in the separate parts of the formulation. The dimensions of the polypill were selected according to the drug loading in respect of selected excipients $(5.85 \mathrm{~mm}$ (height) $\times 6$ $\mathrm{mm}$ (radius)). The geometry of the polypill was designed using a 3D drawing package (BioCAD, 
121 described in the experimental set up were adapted from clinical studies based on assessment of effect 122 combination therapy on healthy middle aged individuals with one or more risk factors [8].

123

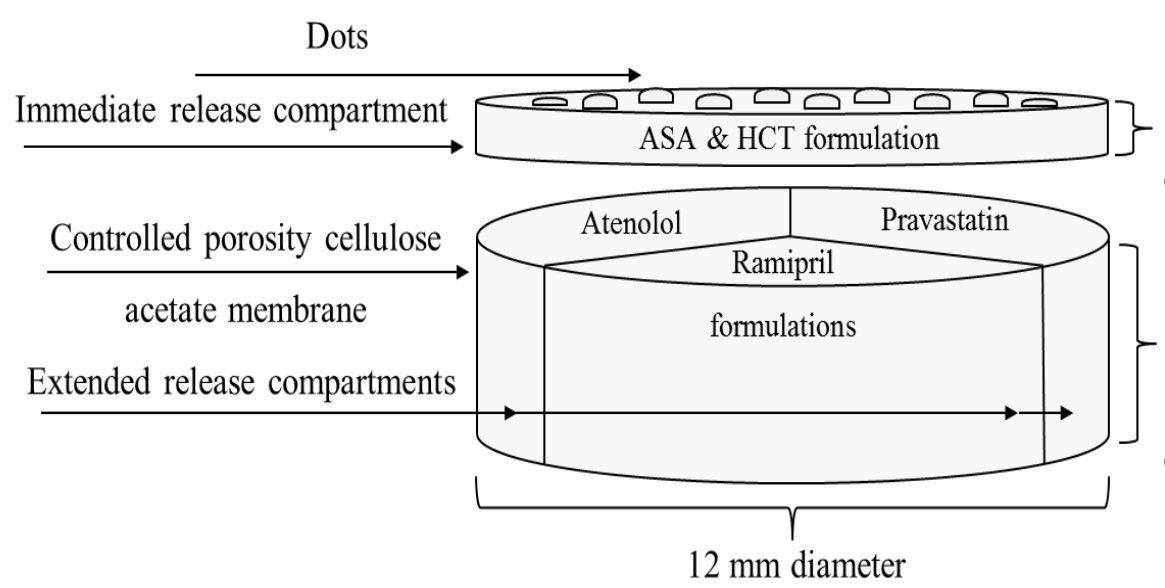

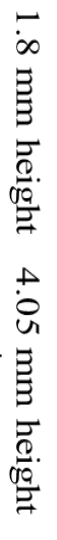

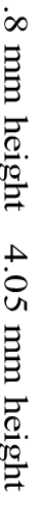

124 Fig. 1. Schematic structural diagram of the polypill design, showing the aspirin and 125 hydrochlorothiazide immediate release compartment and atenolol, pravastatin, and ramipril sustained release compartments.

\subsubsection{Materials for printing of polypill}

128 All powders were mixed using a mortar and pestle for 15 minutes. The printable paste used to form the barrier for the sustained release actives was prepared by mixing $3.15 \mathrm{~g}$ from the blended powder mixture; cellulose acetate (hydrophobic membrane/shell), D-mannitol (a filler), and polyethylene glycol (PEG 6000) (plasticizer) with $1.7 \mathrm{ml}$ of the binder (acetone and dimethyl sulfoxide (DMSO)) until a smooth homogenous paste was achieved according to the formulae in Table 1. DMSO was used to increase the boiling point of the binder system and avoid nozzles blockage due to acetone

134 evaporation (low boiling point) during the extrusion process. We used acetone/DMSO at a ratio of 3:1 v/v. The volume of DMSO per tablet was approximately $28 \mu$ l, equivalent to $30.8 \mathrm{mg}$ (3080 ppm) which is considered a very small volume/quantity in respect to DMSO human toxicity and was within the limit of the class 3 solvents (5000 ppm/day) which stated in ICH Harmonised Tripartite Guidance [27-31]. 
139 Table 1. The weight percentage composition of various ingredients in cellulose acetate shell for 140 sustained release formulation in feed stock.

141

142

143

144

\begin{tabular}{ccc}
\hline Ingredients & Function & Coating $(\% \mathrm{w} / \mathrm{w})$ \\
\hline Cellulose acetate & Hydrophobic shell & 22.64 \\
D-mannitol & Filler & 62.26 \\
PEG (6000) & Plasticizer & 15.10 \\
\hline
\end{tabular}

145 Powders of atenolol, pravastatin, and ramipril were separately blended using a mortar and pestle for

$14615 \mathrm{~min}$ with the required excipients, to ensure homogeneous powder blend. Ultra-pure water was

147 added to the powder and mixed as above method according to the formulae shown in Table 2 . The

148 immediate release layer was composed of aspirin and hydrochlorothiazide (active ingredients),

149 sodium starch glycolate (disintegrant), and polyvinylpyrrolidone (PVP K30) (binder). The powder

150 was blended and mixed with ultra-pure water to form a smooth and soft paste according to the

151 formulae shown in Table 3.

152 Table 2. The weight percentage composition of various ingredients in atenolol, pravastatin, and ramipril formulation feed stock for the sustained release compartments of the polypill.

Ingredients Function $\quad$ ATEN-HPMC ${ }^{*}$ PRA-HPMC ${ }^{* *}$ RAM-HPMC ${ }^{* * *}$

156

157

158

159

160

161

162

$(15 \% \mathrm{w} / \mathrm{w}) \quad(15 \% \mathrm{w} / \mathrm{w}) \quad(15 \% \mathrm{w} / \mathrm{w})$

\begin{tabular}{ccccc}
\hline Atenolol & Active ingredient I & 30.00 & ---- & - --- \\
Pravastatin & Active ingredient II & ---- & 20.00 & ---- \\
Ramipril & Active ingredient III & ---- & ---- & 15.00 \\
HPMC 2208 & Hydrophilic matrix & 15.00 & 15.00 & 15.00 \\
Lactose & Filler & 55.00 & 65.00 & 70.00
\end{tabular}

${ }^{*} \mathrm{ATEN}=$ atenolol,${ }^{* *} \mathrm{PRA}=$ pravastatin, and ${ }^{* * *} \mathrm{RAM}=$ ramipril 
163
Table 3. The weight percentage composition of various ingredients in aspirin and hydrochlorothiazide immediate release formulation feed stock for the immediate-release compartment of the polypill.

Ingredients Function $\quad$ ASA_HCT-IR ${ }^{*}$ compartment

$\begin{array}{ccc}\text { Hydrochlorothiazide } & \text { Active ingredient } & 5.86 \\ \text { Sodium starch glycolate } & \text { Disintegrant } & 55.18 \\ \text { Polyvinylpyrollidine K30 } & \text { Binder } & 10.34\end{array}$

${ }^{*} \mathrm{ASA}=$ acetylsalicylic acid (aspirin), $\mathrm{HCT}=$ hydrochlorothiazide, and IR = immediate release

\subsection{3. $3 D$ based extrusion printing process}

All the pastes were loaded into separate ink cartridges for extrusion through a $500 \mu \mathrm{m}$ print tip. The cellulose acetate shell was first extruded (no drying step needed), followed by extrusion of sustained release pastes inside the segmented cellulose acetate shell. The immediate release layer was then extruded on the top to cover the sustained release compartments, with the addition of identifying raised dots to illustrate the ability to provide integrated identification (visual and feel based) of the polypill (Fig. 2). The total printing time was $25 \mathrm{~min}$ followed by being placed in a vacuum dryer at 40 ${ }^{\circ} \mathrm{C}$ for 24 hours for complete drying.
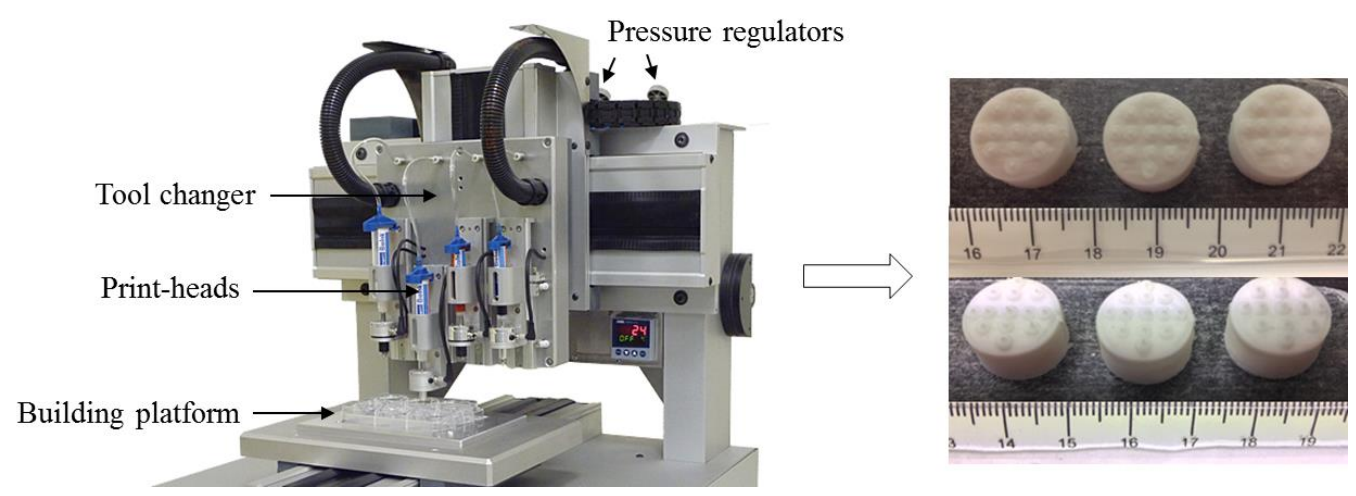

Fig. 2. Photograph of regenHU 3D printer (left) [32], and image of multi-active tablet (right) (5.85 $\mathrm{mm}$ (height) $\times 6 \mathrm{~mm}$ (radius) composed of sustained release compartments (bottom), and immediate release dotted compartment (top). 
183

184

185

186

187

188

189

190

191

192

193

194

195

196

197

198

199

200

201

202

203

204

205

206

207

\subsubsection{Dissolution studies}

A HP Agilent 1050 HPLC equipped with an ACE C18-AR analytical column (100 mm x 4.6 mm) with $5 \mu \mathrm{m}$ particle size was used to analyse dissolution release media for drug content. The autosampler was set up to make $40 \mu 1$ injections, every 25 minutes. The flow rate of the mobile phase was $1 \mathrm{ml} / \mathrm{min}$, the column temperature was $40{ }^{\circ} \mathrm{C}$ and the UV detection wavelength was $215 \mathrm{~nm}$. The mobile phase (acetonitrile and water containing $0.1 \% \mathrm{v} / \mathrm{v}$ of trifluoroacetic acid) was degassed and filtered through a $0.45 \mu \mathrm{m}$ membrane filter. A mixture of actives $(75 \mathrm{mg}$ of aspirin, $12.5 \mathrm{mg}$ of hydrochlorothiazide, $25 \mathrm{mg}$ of atenolol, $20 \mathrm{mg}$ of pravastatin, and $5 \mathrm{mg}$ of ramipril) was dissolved in the dissolution medium and separated using the above HPLC method (Supplementary data, Appendix A, Fig. S.I. 1).

In vitro drug release studies of the 3D printed polypill were performed using USP Type I apparatus (rotation speed at $50 \mathrm{rpm}, 900 \mathrm{ml}$ phosphate buffer, $\mathrm{pH} 6.8$ containing $0.5 \%$ of tween $80(\mathrm{v} / \mathrm{v})$ as the dissolution media at $37^{\circ} \mathrm{C} \pm 0.5^{\circ} \mathrm{C}$ ). $5.0 \mathrm{ml}$ samples were withdrawn at $5,15,30,60,120,240,360$, $480,600,720 \mathrm{~min}$. The samples were centrifuged and a small volume from the supernatant was drawn and filled into HPLC amber glass vials. The samples were kept at $4{ }^{\circ} \mathrm{C}$ (to decrease drug degradation) until tested. Drug dissolution studies were conducted in triplicate and the average of percentage of cumulative drug release as a function of time was determined.

\subsubsection{X-Ray Powder Diffraction (XRPD)}

The XRPD patterns of pure atenolol, pravastatin, ramipril, hydrochlorothiazide, and aspirin and their formulations (immediate and sustained release formulations) were obtained at room temperature using an X'Pert PRO (PANalytical, Almelo, Netherlands) setup in reflection mode using $\mathrm{Cu} \mathrm{K} \alpha_{1}$ (lambda $=$ $1.54 \AA$ A) operating in Bragg-Brentano geometry. The generator voltage was set to $40 \mathrm{kV}$ and the current to $40 \mathrm{~mA}$ and the samples were scanned over $2 \theta$ range of $5^{\circ}$ until $30^{\circ}$ in a step size of $0.026^{\circ}$. 
209 In order to investigate possible interactions between the actives and the selected excipients in their

210 formulations, infrared spectra of pure active ingredients and their formulations were obtained using an

211 ATR-FTIR (Agilent Cary 630 FTIR) spectrometer.

\section{3. Results and discussion}

\section{3.1. In vitro drug dissolution}

214 Dissolution data from the polypill (Fig. 3) show that more than $75 \%$ of the aspirin and

215 hydrochlorothiazide were released within the first 30 minutes. This drug release is attributed to the 216 inclusion of the disintegrant, sodium starch glycolate, which rapidly absorbs water and swells leading 217 to rapid disintegration of this portion of the polypill and fast drug release. The same figure also shows 218 that atenolol, pravastatin, and ramipril displayed sustained release over a period of $720 \mathrm{~min}$ as 219 required; with $69 \%, 81 \%$, and $66 \%$ released respectively. This release is consistent with the effects 220 of rapid hydration of the HPMC leading to a gel like formation and swelling to form a hydrophilic 221 matrix that slows drug release and also to the presence of the permeable cellulose acetate shell which 222 is expected to retard drug release [33-35].

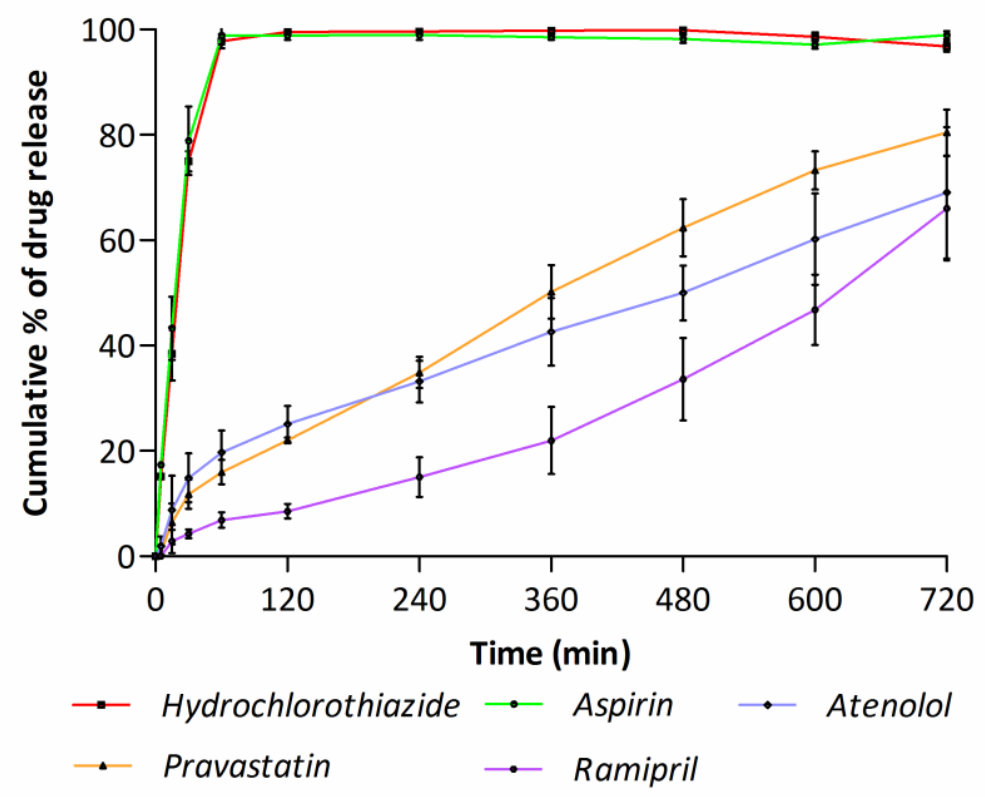


224 Fig. 3. In vitro cumulative drug release profile of each drug from the five drug-loaded compartments

225 of the polypill.

226 These data clearly illustrate the potential to achieve different drug release profiles from the same

227 tablet for different drugs. As the three drugs that require sustained release are in separate 228 compartments this also clearly shows the opportunity that 3D printing provides to vary loading and 229 the fine detail of each drug release.

\subsection{Physical characterisation of immediate and sustained release formulations}

231

\subsubsection{XRPD}

232

XRPD data were collected on the pure as-received drugs before printing, and on the mixed formulations (immediate and sustained release formulations) containing the drugs after printing, in order to investigate any changes in physical form on printing (Figs. 4 and 5). All as-received materials exhibit multiple sharp Bragg peaks in their XRPD patterns related to their crystalline nature, except pravastatin which exhibits no sharp Bragg peaks indicating that it is exist in an amorphous state. The patterns for the crystalline materials match those reported in the Cambridge Structural Database (Fig. 4) [36-39]. After formulation and printing, the Bragg peaks for ramipril, aspirin, hydrochlorothiazide, and atenolol are still present, with peaks also observed as expected from the excipients. For example, the appearance of a sharp peaks due to lactose are clearly visible at 20,19, and 18 degrees 2-theta for

241 the pravastatin, ramipril, and atenolol formulations, and the broad feature due to HPMC is visible at 242 around 20 degrees 2-theta for the atenolol formulation. There is therefore no evidence of a change in 243 physical form for the drugs in these three formulations. The situation is comparable for pravastatin, 244 for which no obvious Bragg peaks from pravastatin are visible in both as-received and in the final 245 formulation. 

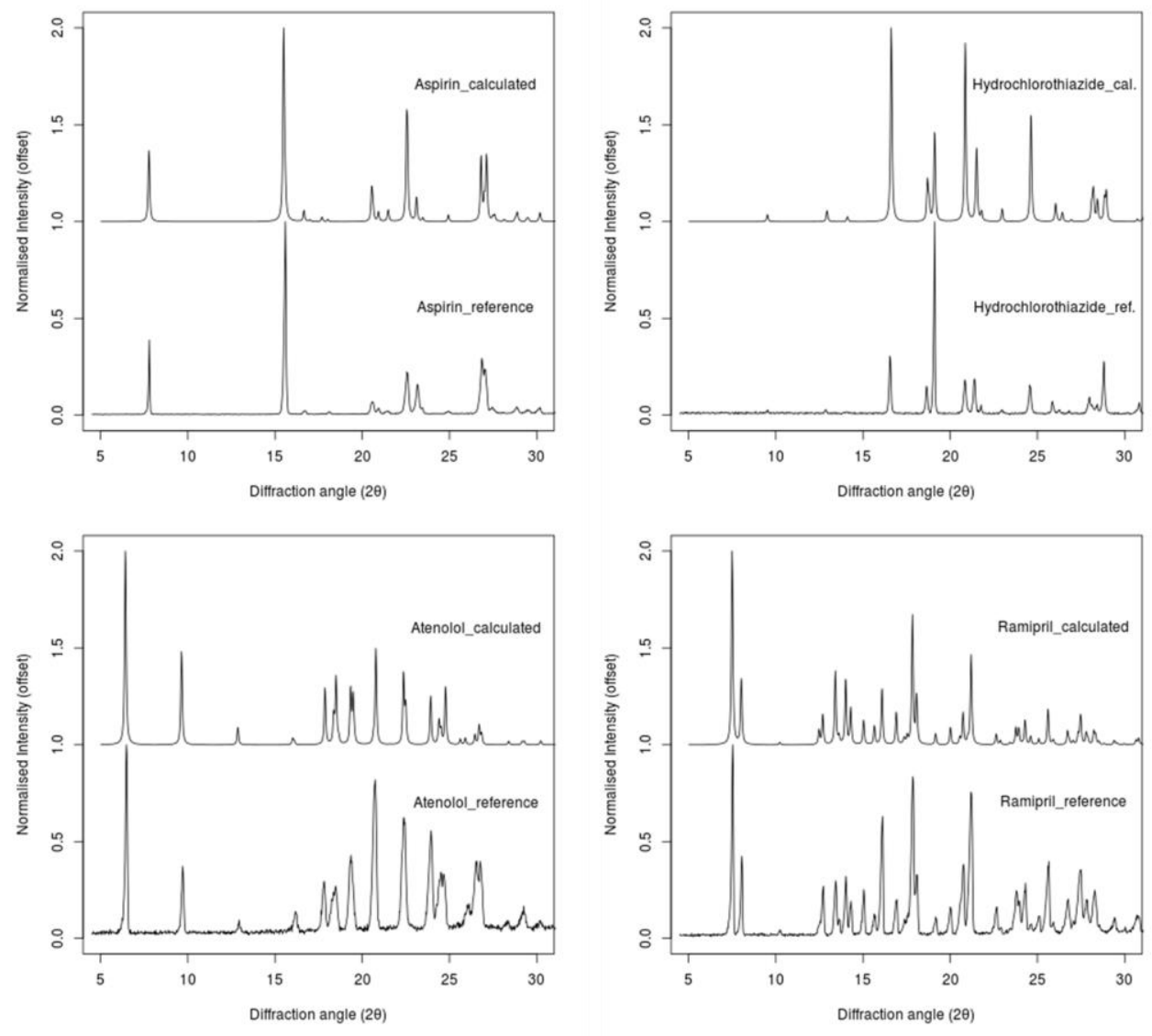

247 Fig. 4. XRPD patterns of the calculated and reference (measured) aspirin (top-left), 248 hydrochlorothiazide (top-right), atenolol (bottom-left), and ramipril (bottom-right). 

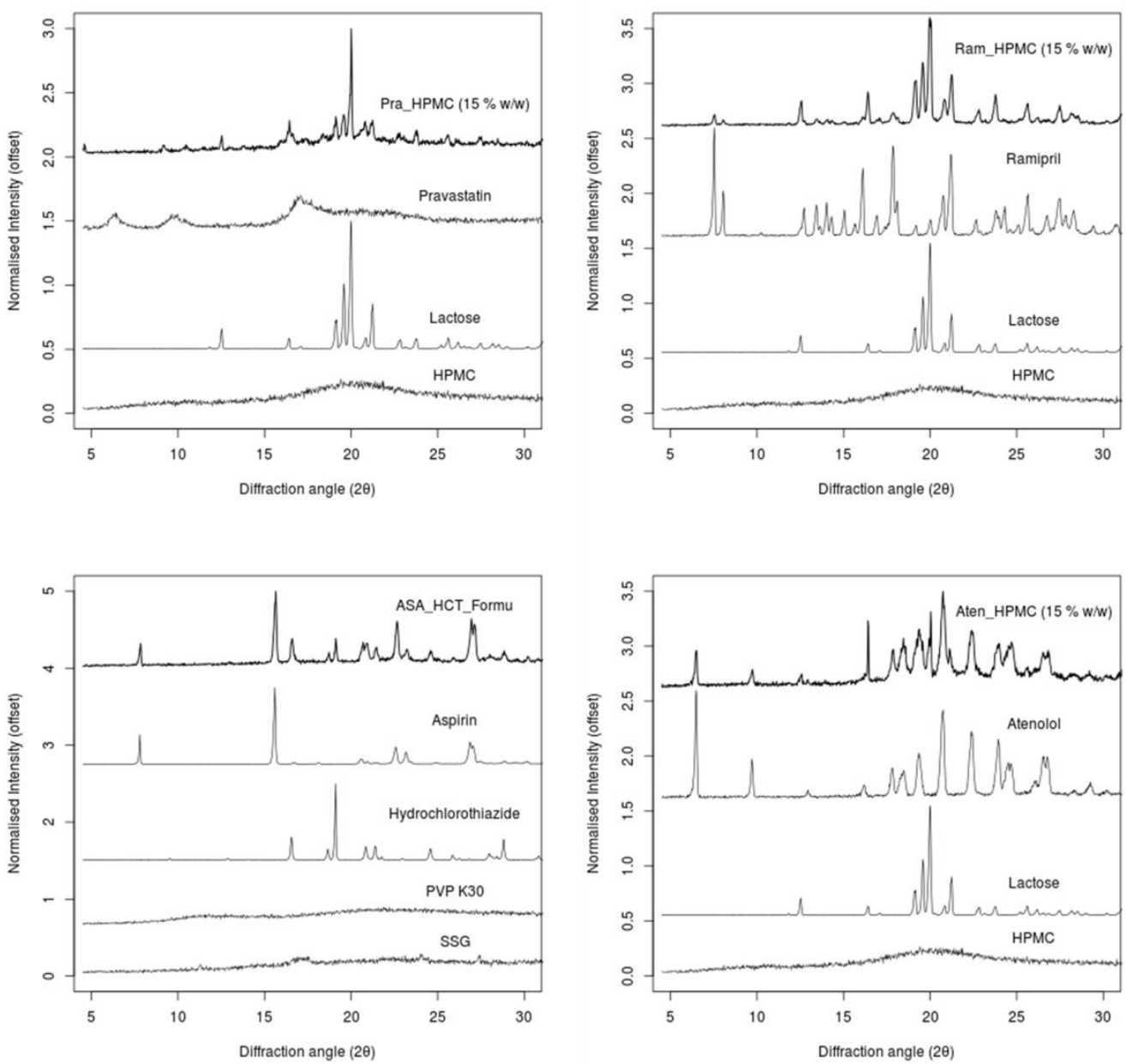

250 Fig. 5. XRPD patterns of $\operatorname{Pra-HPMC}(15 \% \mathrm{w} / \mathrm{w})$, pure pravastatin, lactose, and HPMC (top-left), 251 Ram-HPMC (15\% w/w), pure ramipril, lactose, and HPMC (top-right), ASA-HCT-Formu, pure 252 aspirin, pure hydrochlorothiazide, (polyvinylpyrrolidine) PVP k30, and sodium starch glycolate 253 (bottom-left), and Aten-HPMC (15\% w/w), pure atenolol, lactose, and HPMC (bottom-right).

\subsubsection{ATR-FTIR}

255 The major diagnostic infrared peaks of the actives did not change within the formulations as 256 compared to control spectra of the drugs alone, indicating that there were no detectable interactions 257 between the drugs and the selected excipients (Fig 6). 

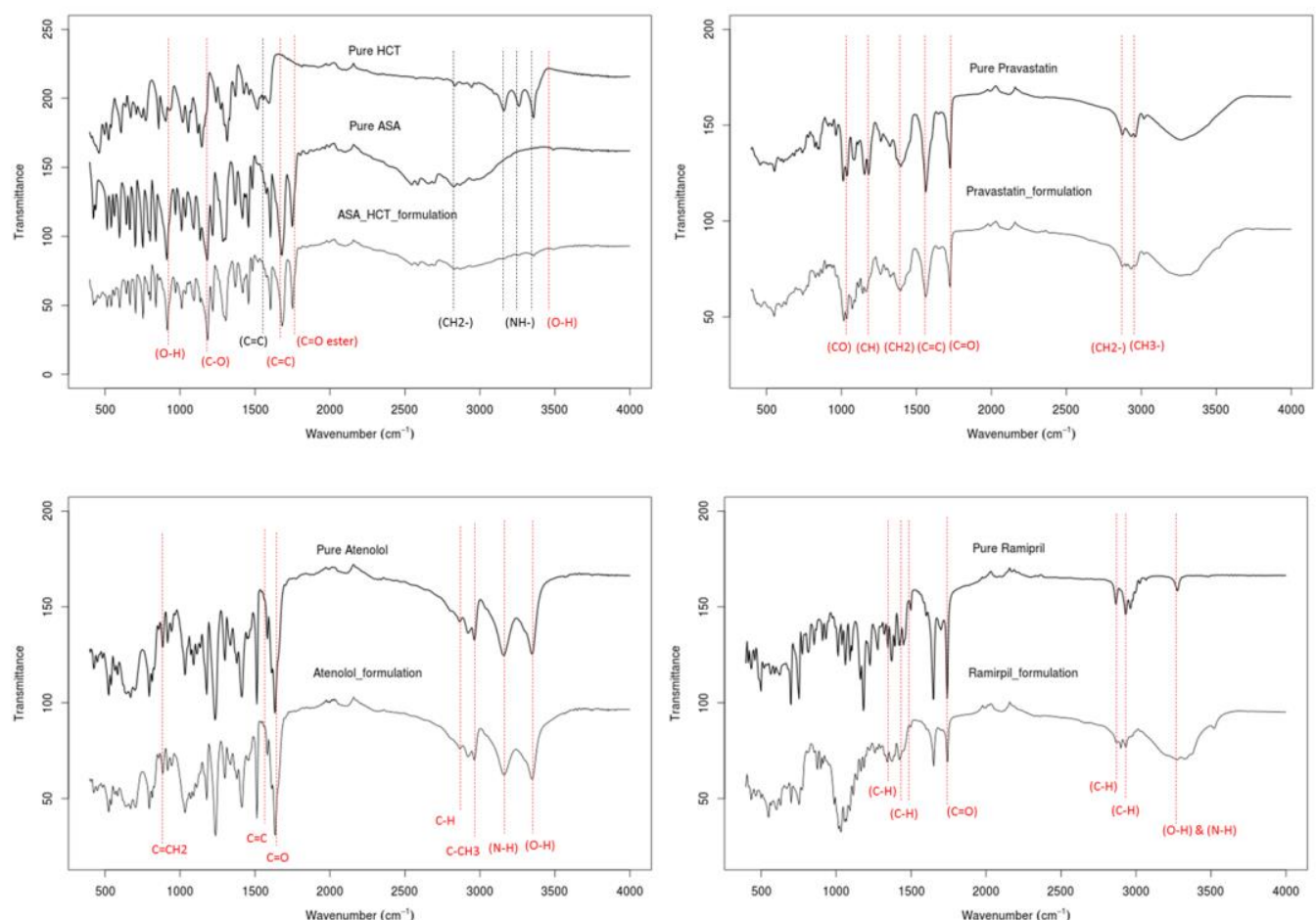

259 Fig. 6. FTIR spectra of pure actives; aspirin and hydrochlorothiazide (top left), pravastatin (top right), atenolol (bottom left), and ramirpil (bottom right) and their formulations.

261 It should be noted that all the polypills were printed to the expected size, mechanically stable, and can be handled readily without any loss of structural integrity.

A relationship has been shown between heart disease and renal failure and various modifiable risk factors, such as hypertension, dyslipidaemia, and platelet capacity [40-43]. Therefore, patients over 55 years old with one or more risk factors, including hypertension, obesity, and diabetes can benefit from a combined medications such as that demonstrated here that includes an antiplatelet, cholesterol and blood pressure lowering agents [44-46]. To use a combined medication approach successfully a number of challenges need to be considered, including; making the novel dosage form acceptable to health professionals and patients; formulation issues; additional cost; achieving regulatory approval; ensuring this becomes a first-line therapy (should be more effective and have no more side effects than drugs taken individually) [3]. The polypill demonstrated here addresses some of these issues, it is of an acceptable size and appearance for a patient, offers the prospect of increasing patient adherence 
273 since only one tablet needs to be taken [4], and also should reduce risk of mistakes by patients who

274 forget to take a certain medicine within a combination of different tablets [3]. Issues such as cost and

275 regulatory approval are beyond the scope of the current work.

276 Furthermore, application of 3D printing in pharmaceutics could offer a flexible tool to tailor the 277 combined drug doses according to the patient's needs. For example, control of tablet size and shape 278 for children and elderly patient with difficulty handling tablets or swallowing, and printing 'specials' 279 for tablets with allergies to certain excipients. We have demonstrated in this paper that a simple 280 visual/tactile identifier can readily be added to 3D printed tablets to aid sight-compromised patient as 281 well.

\section{4. Conclusions}

283 We have successfully demonstrated 3D extrusion printing of a novel complex geometry five-in-one polypill. We have also proved that the polypill is able to deliver five actives via two different and well defined release mechanisms: immediate and sustained release. The drugs were physically separated in the polypill to avoid incompatibility issues and allow maximum flexibility in manipulating the environment of each drug. XRPD and FTIR data were used to show that there was no detectable interaction between the drugs and the chosen excipients, and that our method of 3D printing did not lead to a detectable change in the physical form of the drugs (e.g. polymorphism, hydration etc). Such a combination of actives as used here has been shown to be important in prevention and treatment of cardiovascular diseases. The drugs combined as a polypill provide the prospect of improved adherence to such combination therapies due to the convenience of a single tablet and the potential to optimise and personalise dosages and release for each drug independently in such multi-drug dosage 294 forms.

\section{Acknowledgments}

296 We gratefully acknowledge Libyan government for the project grant to support a $\mathrm{PhD}$ studentship for

297 SAK. We thank DFE Pharma for the complementary supply of sodium starch glycolate and Paul 
298 Cooling, Colin Wills and Michael Wallis for technical assistance. Maureen Alexander is thanked for 299 inspiring the interest of MRA in the application of 3D printing in polypharmacy.

300 


\section{References}

302

303

304

305

306

307

308

309

310

311

312

313

314

315

316

317

318

319

320

321

322

323

324

325

326

327

[1] J. Gao, C. Chen, J.-X. Chen, L.-M. Wen, G.-L. Yang, F.-P. Duan, Z.-Y. Huang, D.-F. Li, D.-R. Yu, H.-J. Yang, S.-J. Li, Synergism and Rules of the new Combination drug Yiqijiedu Formulae (YQJD) on Ischemic Stroke based on amino acids (AAs) metabolism, Sci. Rep., 4 (2014) 1-11.

[2] S. Bangalore, A. Shahane, S. Parkar, F.H. Messerli, Compliance and fixed-dose combination therapy, Curr. Hypertens. Rep., 9 (2007) 184-189.

[3] P. Sleight, H. Pouleur, F. Zannad, Benefits, challenges, and registerability of the polypill, Eur. Heart J., 27 (2006) 1651-1656.

[4] S.A. Khaled, J.C. Burley, M.R. Alexander, C.J. Roberts, Desktop 3D printing of controlled release pharmaceutical bilayer tablets, Int. J. Pharm., 461 (2014) 105-111.

[5] M. Lafeber, D.E. Grobbee, M.L. Bots, S. Thom, R. Webster, A. Rodgers, F.L. Visseren, W. Spiering, The Evening versus Morning Polypill Utilization Study: the TEMPUS rationale and design, Eur. J. Prev. Cardiol., 4 (2013) 425-433.

[6] J.D. Spence, Polypill: for Pollyanna*, Int. J. Stroke, 3 (2008) 92-97.

[7] K.M. Carey, M.R. Comee, J.L. Donovan, A.O. Kanaan, A polypill for all? Critical review of the polypill literature for primary prevention of cardiovascular disease and stroke, Ann. Pharmacother., 46 (2012) 688-695.

[8] S. Yusuf, P. Pais, R. Afzal, D. Xavier, K. Teo, J. Eikelboom, A. Sigamani, V. Mohan, R. Gupta, N. Thomas, Effects of a polypill (Polycap) on risk factors in middle-aged individuals without cardiovascular disease (TIPS): a phase II, double-blind, randomised trial, Lancet, 373 (2009) 13411351.

[9] J. Hippisley-Cox, C. Coupland, Effect of combinations of drugs on all cause mortality in patients with ischaemic heart disease: nested case-control analysis, BMJ, 330 (2005) 1059-1063.

[10] C.P. Limited. (2015). Polycap Retrieved 26/06/2015, from http://www.polycap.org/index.html [11] D.M. Lloyd-Jones, E.P. Leip, M.G. Larson, R.B. d'Agostino, A. Beiser, P.W. Wilson, P.A. Wolf, D. Levy, Prediction of lifetime risk for cardiovascular disease by risk factor burden at 50 years of age, Circulation, 113 (2006) 791-798. 

cardiovascular diseases: key concepts, current status, challenges, and future directions, Circulation, 122 (2010) 2078-2088.

[13] J. Jia, C. Dong, W. Zhang, Y. Cui, J. Liu, Evaluation of pharmacokinetic and pharmacodynamic relationship for oral sustained-release atenolol pellets in rats, J. Pharm. Biomed. Anal., 55 (2011) 342348.

[14] N.A. VH, G. Niharika, P. Deepak, S. Nazan, S.A. Mohammed, Formulation design, characterisation and in vitro evaluation of bilayered tablets containing telmisartan and hydrochlorthizide, Int. J. Bio., 1 (2013) 1-9.

[15] S. Shafiq, F. Shakeel, S. Talegaonkar, F.J. Ahmad, R.K. Khar, M. Ali, Development and bioavailability assessment of ramipril nanoemulsion formulation, Eur. J. Pharm. Biopharm., 66 (2007) 227-243.

[16] A.T. Cohen, S. Imfeld, J. Markham, S. Granziera, The use of aspirin for primary and secondary prevention in venous thromboembolism and other cardiovascular disorders, Thromb. Res. , 135 (2015) 217-225.

[17] S.C. Halbert, B. French, R.Y. Gordon, J.T. Farrar, K. Schmitz, P.B. Morris, P.D. Thompson, D.J. Rader, D.J. Becker, Tolerability of Red Yeast Rice (2,400 mg Twice Daily) Versus Pravastatin (20 mg Twice Daily) in Patients With Previous Statin Intolerance, Am. J. Cardiol., 105 (2010) 198-204. [18] W. Liu, Y. Li, J. Liu, X. Niu, Y. Wang, D. Li, Application and Performance of 3D Printing in 347 Nanobiomaterials, J. Nanomater., 2013 (2013) 1-7.

348 [19] J. Skowyra, K. Pietrzak, M.A. Alhnan, Fabrication of extended-release patient-tailored 349 prednisolone tablets via fused deposition modelling (FDM) 3D printing, Eur. J. Pharm. Sci. , 68 $350 \quad$ (2015) 11-17.

[20] C.L. Ventola, Medical Applications for 3D Printing: Current and Projected Uses, Pharm. Ther., 39 (2014) 704-711.

353 [21] N. Scoutaris, M. Snowden, D. Douroumis, Taste masked thin films printed by jet dispensing, Int. 354 J. Pharm., (In press 2015). 

analysis of chemical heterogenities in inkjet micro-array printed drug/polymer formulations, J. Mater.

[23] N. Scoutaris, M.R. Alexander, P.R. Gellert, C.J. Roberts, Inkjet printing as a novel medicine formulation technique, J. Control. Release, 156 (2011) 179-185.

[24] A. Goyanes, A.B.M. Buanz, G.B. Hatton, S. Gaisford, A.W. Basit, 3D printing of modifiedrelease aminosalicylate (4-ASA and 5-ASA) tablets, Eur. J. Pharm. Biopharm., 89 (2015) 157-162. oral dosage forms fabricated by three dimensional printing ${ }^{\mathrm{TM}}$, J. Control.Release, 66 (2000) 11-17.

[26] W.E. Katstra, R.D. Palazzolo, C.W. Rowe, B. Giritlioglu, P. Teung, M.J. Cima, Oral dosage 497-506.

[28] S.W. Jacob, C. Jack, Dimethyl Sulfoxide (DMSO) in Trauma and Disease, CRC Press, Florida, 2015.

[29] H. Changqin, L. Ying, Quality Control in Pharmaceuticals: Residual Solvents Testing and Veterinarian, 46 (1984) 89-95.

374 [31] International Conference on Harmonization of Technical Requirements for the Registration of 375 Pharmaceuticals for Human Use, Q3C (R4) Impurities: Guideline for Residual Solvents, 2009.

376 [32] RegenHU. (2015). 3D Discovery ${ }^{\circledR}$ instrument, 3D Bio-Printer [Photograph] Retrieved April 04 2015, from http://www.regenhu.com/products/3d-bio-printing.html (2013) 883-901. 
382 Controlled Release: A Historical Perspective, in: R.P. Samuel, T. Peter, D.M. Colin (Eds.) 383 Hydrophilic Matrix Tablets for Oral Controlled Release, Springer, New York, 2014, pp. 17-51.

384

[35] S.A. Khaled, J.C. Burley, M.R. Alexander, J. Yang, C.J. Roberts, 3D printing of tablets containing multiple drugs with defined release profiles, Int. J. Pharm., (In Press 2015).

[36] C.C. Wilson, Interesting proton behaviour in molecular structures. Variable temperature neutron diffraction and ab initio study of acetylsalicylic acid: characterising librational motions and comparing protons in different hydrogen bonding potentials, New J. Chem., 26 (2002) 1733-1739.

[37] R. Esteves de Castro, J. Canotilho, R.M. Barbosa, M.R. Silva, A.M. Beja, J. Paixao, J.S. Redinha, Conformational isomorphism of organic crystals: racemic and homochiral Atenolol, Cryst. Growth Des., 7 (2007) 496-500.

[38] N. Nagel, H. Schweitzer, H. Urbach, W. Heyse, B. Müller, H. Berchtold, Ramipril, Acta Cryst. Section B, 57 (2001) 463-465.

[39] L. Dupont, O. Dideberg, Structure cristalline de l'hydrochlorothiazide, C7H8CIN3O4S2, Acta Cryst. Section B, 28 (1972) 2340-2347.

[40] P.S. Collaboration, Age-specific relevance of usual blood pressure to vascular mortality: a metaanalysis of individual data for one million adults in 61 prospective studies, Lancet, 360 (2002) 19031913.

[41] T. Pedersen, Randomised trial of cholesterol lowering in 4444 patients with coronary heart disease: the Scandinavian Simvastatin Survival Study (4S), Atheroscler. Suppl. , 5 (2004) 81-87.

[42] JAMA Network Webcasts, Executive summary of the third report of the National Cholesterol Education Program (NCEP) expert panel on Detection, Evaluation, and Treatment of high blood cholesterol in adults (Adult Treatment Panel III), JAMA, 285 (2001) 2486-2497.

[43] Antithrombotic Trialists' Collaboration, Collaborative meta-analysis of randomised trials of antiplatelet therapy for prevention of death, myocardial infarction, and stroke in high risk patients, BMJ, 324 (2002) 71-86. 
407 [44] N.J. Wald, M.R. Law, A strategy to reduce cardiovascular disease by more than $80 \%, B M J, 326$ 408 (2003) 1419.

409 [45] M. Law, N. Wald, J. Morris, R. Jordan, Value of low dose combination treatment with blood 410 pressure lowering drugs: analysis of 354 randomised trials, BMJ, 326 (2003) 1427.

411 [46] M.R. Law, N.J. Wald, A. Rudnicka, Quantifying effect of statins on low density lipoprotein 412 cholesterol, ischaemic heart disease, and stroke: systematic review and meta-analysis, BMJ, 326 413 (2003) 1423.

414

415 